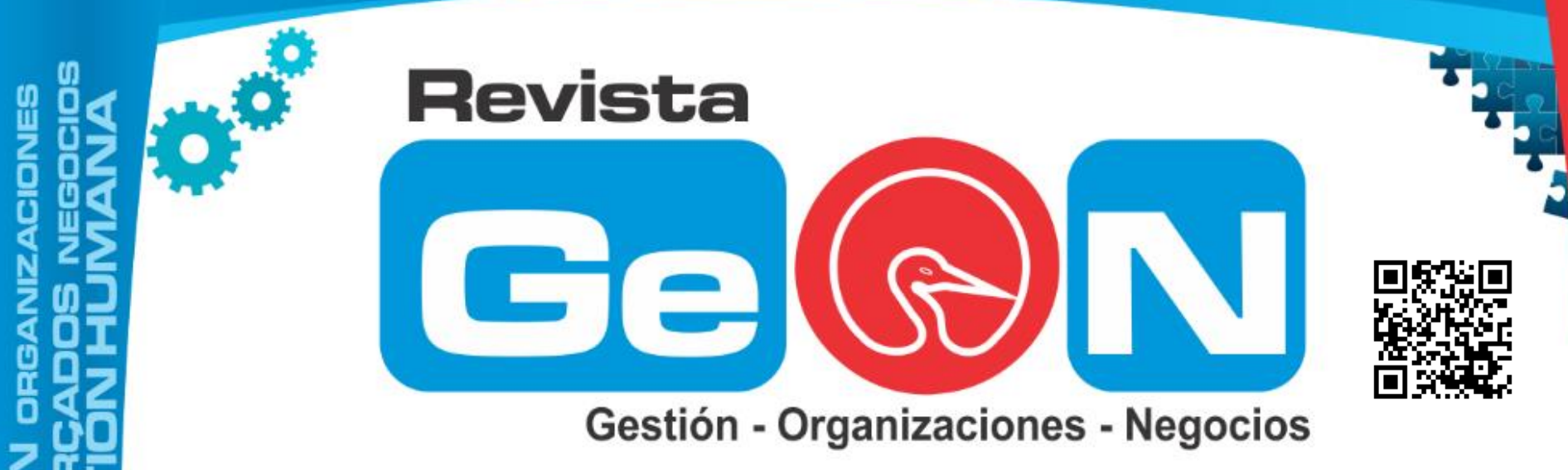

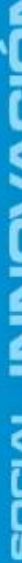

(1)

ISEN 204E - 3970

Volumen 11 Número 0 Diciembre Jullo 14

Revista Electrónica de la Facultad de Ciencias Económicas de la Universidad de los Llanos

$4 \leq 1$

$3<$

$2 \frac{1}{2} \frac{1}{4}$
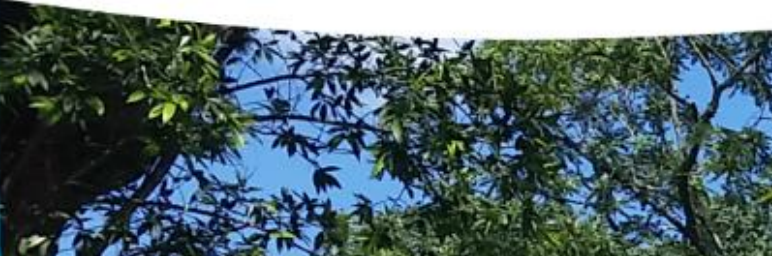

(1)

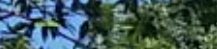

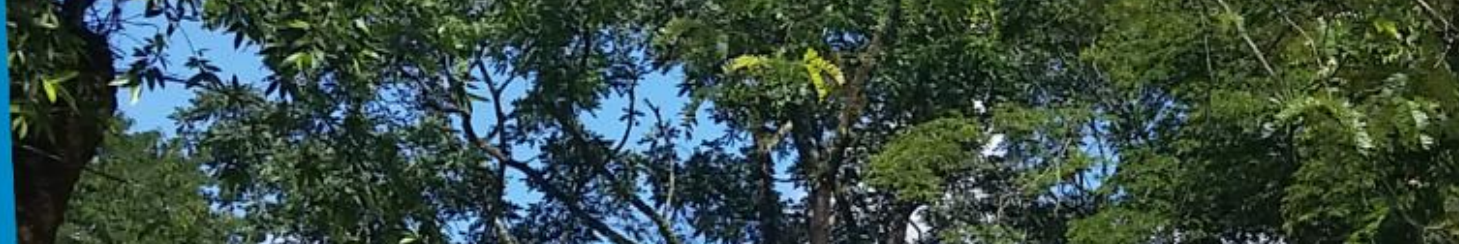

다

(11) को

$2 \frac{1}{0}$

i c

iii $\frac{2}{11}$ if

in

402

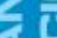

40

20 III

iㅣㄴ

12

10

10

ii 1

U1

$2 \frac{1}{2}$

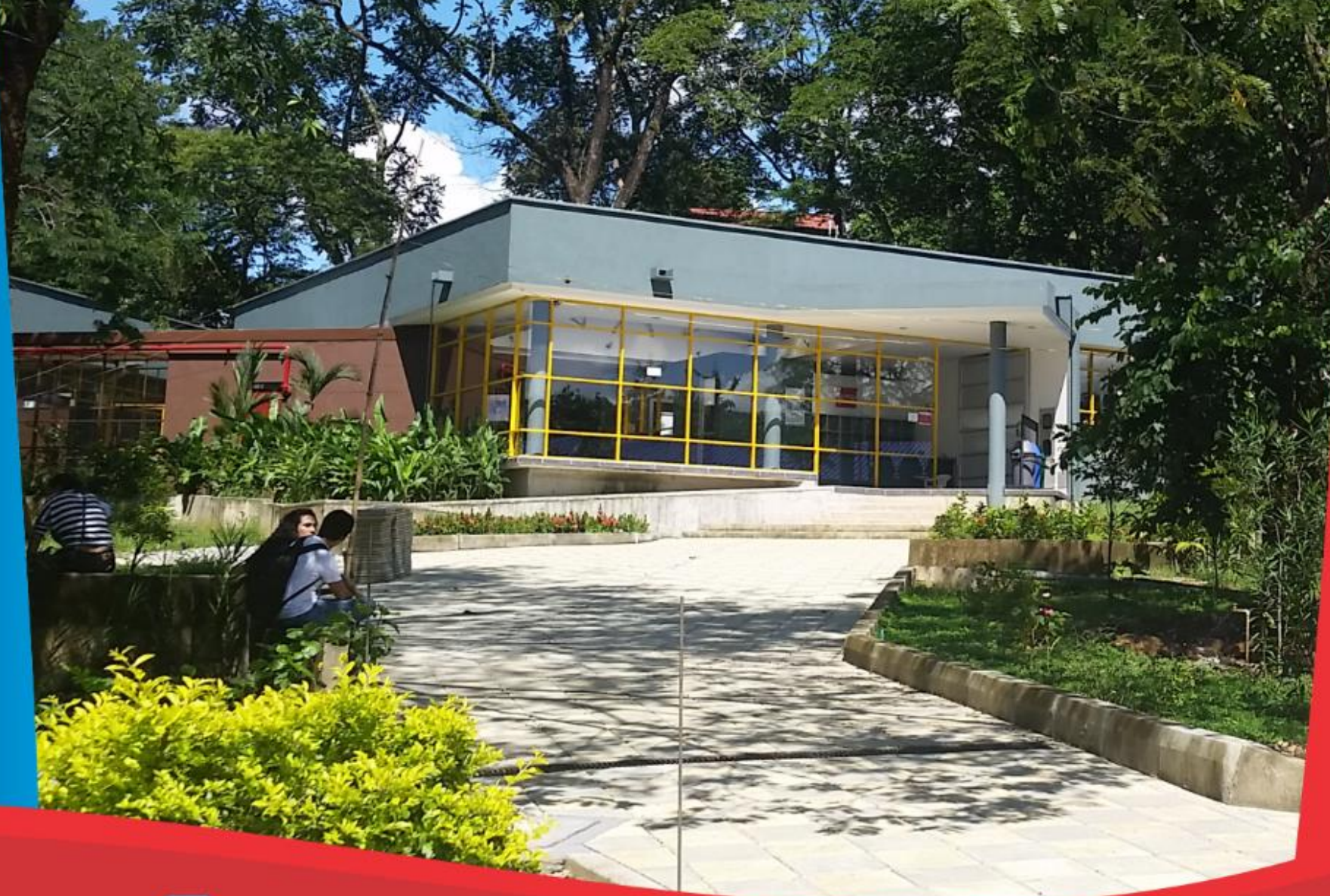

I/revistaGEON @RevistaGeon

http://revistageon.unillanos.edu.co 


\section{Retos y Oportunidades Para Las Empresas Del Meta Frente A La Alianza Pacífico - por Juan Carlos Leal Céspedes}

Juan Carlos Leal Céspedes

Administrador de Empresas, Especialista en Alta Gerencia, Candidato a Magister MBA

Universidad Nacional de Manizales

@jklealc

Resultado del Grupo de Estudio G\&DO de la Facultad de Ciencias Económicas de la Universidad de los Llanos

El 07 de agosto de 1990, el Dr. Cesar Gaviria Trujillo, tomó posesión como Presidente de la República de Colombia, y en sus apartes del discurso pronunció claramente la frase "Bienvenidos al futuro", y explicó el nuevo escenario económico que el país tenía en frente así, "la apertura es eso: un proceso dinámico de modernización apoyado por el crecimiento de las exportaciones y destinado a garantizarnos un puesto en el mercado mundial. Exportar más, importar más, producir más, hacer más rica nuestra economía, y así generar más empleo. (El Espectador, 1990). De esta manera comunicaba a los colombianos la realidad en la que país debía integrar y proyectar sus esfuerzos para un desarrollo futuro. Desde este momento el país inicia el abandono paulatino de un modelo económico proteccionista e inicia el camino a un modelo de libre comercio.

Aunque el país inició el proceso de acuerdos comerciales, con el objetivo de garantizar el crecimiento económico, busco acuerdos con sus socios más importantes, desde 1969 al ingresar a la Comunidad Andina (CAN), posteriormente al Grupo de los tres (G3) en 1989, surge el apoyo de Estados Unidos a través de la Ley de preferencias arancelarias aduaneras (ATPA) en 1991; el establecimiento e integración del Área de libre comercio (ALCA) en 1994. Por ser miembro de la CAN, obtiene ingreso a MERCOSUR, posteriormente integra el Área de libreo comercio del Caribe (CARICOM) (Republica, 2013). Después del año 2000, dificultades de acuerdos como con Venezuela, o Estados Unidos, obliga al país a guiar su mirada a otros mercados de la región y de Asia.

Desde la década del 90, el país trazó la ruta económica hacia la globalización pero ha sido evidente que el planteamiento de políticas de desarrollo, así como la ejecución de proyectos enfocados al fortalecimiento no ha rendido los mejores frutos.
La oposición de grupos políticos a la decisión estatal de alcanzar una mayor libertad de mercado, lo agrupa Daniel en su libro de Negocios Internacionales, (Daniels J. , 2010) en el que establece las categorías de las razones por las cuales no se acepta la globalización, estas son: amenazas a la soberanía nacional, reflejado en la pérdida de autonomía económica; El crecimiento y estrés ambiental, es poco frente al costo ambiental de los recursos del país; y desigualdad creciente en la distribución del ingreso, lo que ha sido la dificultad más grande por lo que no se refleja en la sociedad colombiana.

Lo ideal para el país es procurar por un equilibrio de los aspectos económicos, es decir, no podemos ser indiferentes al proceso mundial de globalización, por lo que debe gestionar los diferentes mecanismos para integrarse a la nueva realidad, sin desconocer los aspectos fundamentales para el éxito como la inversión en infraestructura, información y generar conocimiento en los diferentes sectores productivos.

El análisis de Eduardo Lora en su artículo de la Revista Dinero, Colombia no ha logrado penetrar los mercados mundiales de productos sofisticados. La sofisticación exportadora es la capacidad de vender en los mercados externos el mismo tipo de bienes que exportan los países más ricos.... El ingrediente principal es la innovación, es decir descubrir continuamente nuevas formas de hacerlo y descubrir nuevas cosas que pueden hacerse bien. (Lora, 2013). Plantea de esta manera que la incursión a nuevos mercados para el país, depende del nivel de diferenciación de sus productos y procesos que realice.

En el 2013, nace la Alianza del pacífico se convierte en uno de los varios tratados que posee Colombia para ampliar los mercados para los productos nacionales. La alianza integrada por México, Perú, Chile y Colombia tiene como principal objetivo genera cadenas de producción y de valor mundial, aprovechando los acuerdos anteriores de cada miembro. Como países observadores están: Portugal, España, Francia, Canadá, Guatemala, El Salvador, Honduras, República Dominicana, Ecuador, Paraguay, Uruguay, Nueva Zelanda, Australia, Japón, Turquía, Corea del Sur, China, Estados Unidos, Panamá y Costa Rica, estos dos últimos candidatos a ser miembros. (pacifico, 2003) 


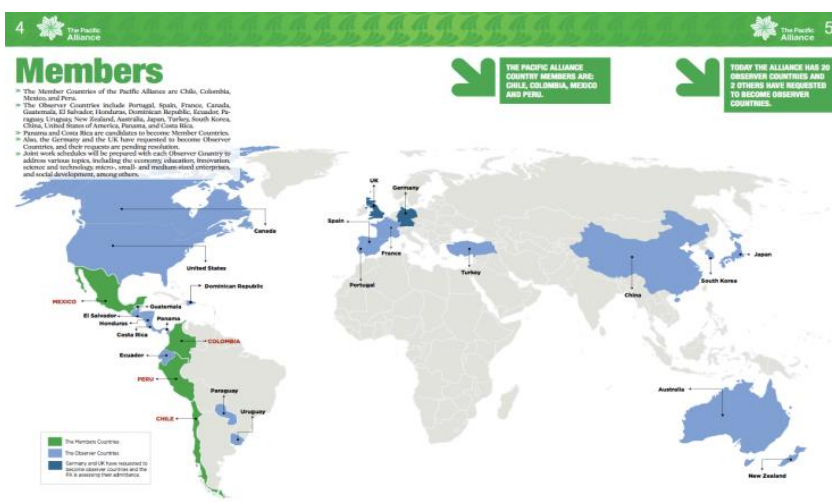

Grafico obtenido de http://alianzapacifico.net

En conjunto de países miembros, poseen una población de 209 millones de personas, cerca al 35\% de la población de América Latina y el Caribe (Pacífico, 2013), con igual porcentaje del PIB latinoamericano, aumentando el PIB per cápita a US\$13.000. La tasa de desempleo de los países miembros fue del 5,8\%, y la tasa media de inflación fue del 3,8\%, inferior a la media del $5,7 \%$ de la Región.

Los pronósticos UNECLA indicar que para 2013, los Países Miembros de la Alianza del Pacífico tendrán un sostenido crecimiento económico promedio de $4,3 \%$, mientras que el crecimiento promedio de la región será del 3,0\%. Además los países miembros ocupan los puestos entre el 37 y 48 del Doing bussines 2012, entre 185 países. Lo anterior plantea la posibilidad y fortaleza económica de la alianza.

A continuación se presenta las posibilidades comerciales de la Alianza, sumando los acuerdos de cada país miembro.

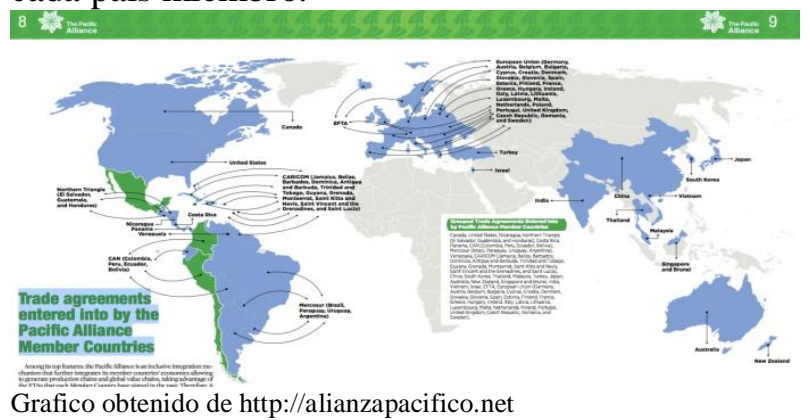

Como logros importantes se tienen los siguientes (Pacífico, 2013):

- Visa eliminación: la eliminación de la visa de turismo para la población de la Alianza para viajar a México.

- La integración del mercado de capitales: Integrado mercado de valores entre Chile, Colombia, Perú y México.
- Embajadas en común: la apertura compartida Embajadas como Ghana, Argelia, Marruecos, y Vietnam.

- La cooperación como herramienta para el desarrollo social y económico.

- Cooperación para la Salud.

- Student Mobility Platform: 100 becas anuales por país: 75 becas de posgrado y 25 scholarships dedicados a doctorados y la movilidad académica de los profesores.

- Oficinas de Promoción: Una oficina de promoción conjunta.

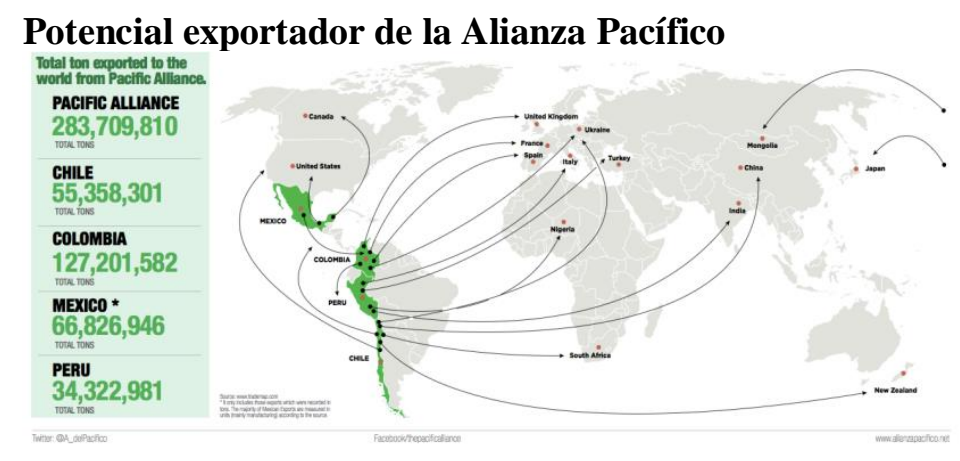

Grafico obtenido de http://alianzapacifico.net

El potencial comercial de los países miembros de la Alianza del Pacífico (Pacífico, 2013) representan un mundo de posibilidades de exportación: la minería, agroindustria, flores, plantas, agrícola, ganadería y acuicultura y los productos pesqueros. En cuanto a los bienes manufacturados, las exportaciones oportunidades incluyen artesanías, envases y embalajes, equipos, cosméticos y artículos de tocador, construcción materiales, electrodomésticos, autopartes y automóviles, aeroespacial, biotecnología, textil y confección, cuero, joyas y baratijas. Y, también hay grandes oportunidades en los servicios, como el turismo y en el campo de la ingeniería, la construcción, la salud, software, animación digital, videojuegos, aplicaciones audiovisuales, entre muchos otros.

El futuro del comercio mundial está en el pacífico, ya que en este hemisferio se sitúa más de la mitad de la población mundial y donde se producen $2 / 3$ partes del PIB total (Duque), por lo tanto la mayor oportunidad se encuentra en la cuenca Pacífico.

Antes de iniciar con la visión general del departamento del Meta, es importante tener en cuenta 
la información generada por el Consejo Privado de Competitividad, integrado por empresas y organismos privados, en donde recopilan información del Banco Mundial, del Word Economics Forum, entre otros.

Teniendo en cuenta solo dos aspectos del informe, (Competitividad, 2012), sobre Ciencia, tecnología e innovación, así como de Infraestructura, transporte y logística, Colombia presenta los siguientes indicadores:

\section{Posición de las empresas del departamento del Meta frente a la Alianza Pacífico}

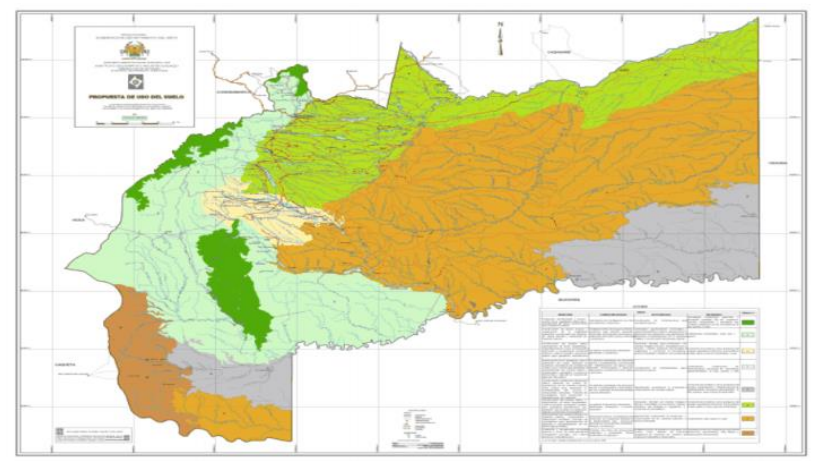

http://www.meta.gov.co/es/el-meta-2/mapas/mapa-de-propuestas-de-usodel-suelo-del-departamento-del-meta/

En Ciencia, tecnología e innovación (Competitividad, 2012), Colombia ocupa el puesto 70 entre 144 países, en donde la inversión en I\&D no ha superado el $0,2 \%$ del PIB del país, y cuya inversión ha sido mayo del sector público que del privado. El panorama actual es que existe interés del gobierno por mejorar este aspecto.

En Infraestructura, transporte y logística (Competitividad, 2012), Colombia ocupa el puesto 104 entre 144 países. El índice de desempeño logístico a 2012 es de 2,9 (5: mejor desempeño, y 1: menor desempeño) otorgando el puesto 64 a nivel mundial

Respecto a la oferta y calidad de la infraestructura (Competitividad, 2012) del país en general es deficiente, teniendo en cuenta las siguientes calificaciones (7: extensiva y eficiente, 1: extremadamente deficiente):

- Vial: 2,7

- Portuario: 1,6

- Férreo: 3,2

- Aeroportuario: 3,8
A continuación se hace una recopilación de indicadores competitivos basados en el diagnóstico de competitividad elaborado por el Consejo Privado de Competitividad 2012-2013.

Informe Nacional de Competitividad 2012 -2013

\begin{tabular}{|c|c|c|}
\hline & Aspecto competitivo & Puesto \\
\hline 1 & Educación superior y formación para el trabajo & $67 / 144$ \\
\hline 2 & $\begin{array}{l}\text { Tasa de mortalidad infantil (entre } 0 \text { y } \\
12 \text { meses) por cada } 1000 \text { nacimientos }\end{array}$ & $79 / 144$ \\
\hline 3 & $\begin{array}{l}\text { Productividad laboral por persona empleada } \\
2010\end{array}$ & 21.827 \\
\hline 4 & Productividad laboral por horas trabajadas & 11 \\
\hline 5 & Eficiencia del mercado laboral & $88 / 144$ \\
\hline 6 & Nivel de Innovación & $70 / 144$ \\
\hline 7 & $\begin{array}{l}\text { Inversión de Colombia en I\&D como \% PIB - } \\
2011\end{array}$ & $0,19 \%$ \\
\hline 8 & $\begin{array}{l}\text { Servicios en línea (de } 0 \text { a } 1 \text { : más servicios } \\
\text { ofrecidos) }\end{array}$ & 0,84 \\
\hline 9 & Interacción electrónica de la ciudadanía & 0,74 \\
\hline 10 & Infraestructura, transporte y logística & $108 / 144$ \\
\hline \multirow[t]{2}{*}{11} & $\begin{array}{l}\text { Desempeño logístico Índice } 2012 \text { (1:menor, 5: } \\
\text { mejor) }\end{array}$ & 2,9 \\
\hline & Oferta de calidad de infraestructura & \\
\hline 12 & $\begin{array}{l}\text { Vial (1: externadamente deficiente, } 7: \\
\text { extensiva y eficiente) }\end{array}$ & 2,6 \\
\hline 13 & Portuario & 1,6 \\
\hline 14 & \begin{tabular}{|l|} 
Férreo \\
\end{tabular} & 3,2 \\
\hline 15 & Aeroportuario & 3,8 \\
\hline 16 & $\begin{array}{l}\text { Costos de exportación e importación (Dólares } \\
\text { por contenedor) }\end{array}$ & $\$ 2.270$ \\
\hline 17 & $\begin{array}{l}\begin{array}{l}\text { Tecnologías de la información } \\
\text { comunicaciones }\end{array} \\
\text { collas } \\
\end{array}$ & $73 / 144$ \\
\hline 18 & Sistema financiero & $67 / 144$ \\
\hline 19 & $\begin{array}{l}\text { Sistema tributario (tarifa total de impuestos } \\
\text { como porcentaje de utilidades, 2011) }\end{array}$ & $136 / 144$ \\
\hline 20 & Eficiencia del mercado de bienes & $99 / 144$ \\
\hline
\end{tabular}

Fuente: (Competitividad, 2012). WEF (World Economics Forum)

Lo anterior no es ajeno a la situación del departamento del Meta, incluso las condiciones que ofrece el departamento son más precarias, teniendo en cuenta que de estos aspectos solo cuenta con vías en buenas condiciones y carece de los demás aspectos.

Actualmente Colombia le apuesta al desarrollo de infraestructura con sus proyectos como: Vía de la prosperidad; Proyectos de hidroeléctricas para la generación de energía; Navegabilidad del Rio Magdalena; y el mejoramiento del Aeropuerto Internacional del Barranquilla "Ernesto Cortissoz". Otros proyectos interesantes han quedado estancados como el proyecto "Eje Tribugá - Urabá", lo que le daría al país salida a los dos océanos (Duque). 
El departamento del Meta (Republica, 2013), con una extensión total de $85.635 \mathrm{Km} 2,29$ municipios y una población proyecta al 2011 (DANE) de 888.802 hab., ocupa el puesto 13 del escalafón de competitividad (CEPAL, 2009), de lo que establece que posee rasgos de categoría "medio alto" en competitividad en aspectos de Gestión de capital humano, así como finanzas y la gestión pública. Calificación "media baja" en aspectos como infraestructura y fortaleza de la Economía, y posee deficiencia con calificación "colero" en aspecto de ciencia y tecnología.

Basados en el Doing Business subnacional (Banco Mundial, 2010), el departamento del Meta se sitúa en el puesto 19 de un total de 21, lo que refleja el grado de complejidad o dificultad para hacer negocios en el Departamento. Los aspectos a mejorar para la realización eficiente de los negocios, de acuerdo a esta evaluación, son: obtención de permisos de construcción, el registro de propiedades y la apertura de empresas. Existe una dificultad media con aspectos como el pago de impuestos y el cumplimiento de los contratos.

El departamento posee una extensa área cultivable, localizada en la Altillanura, antes reconocida como una zona de suelos improductivos (Llanos, 2005), pero que a raíz de los adelantos en investigación agrícola, estos suelos podrían ser acondicionados y explotados de manera sostenible. De esta manera se manifiesta un potencial económico importante para el departamento.

El PIB departamental (departamento, 2013), es del $4,34 \%$ del total nacional con un crecimiento del $11,4 \%$ en los últimos años impulsado por la explotación de hidrocarburos. El PIB del departamento lo conforman principalmente la explotación de minas y canteras con un $58,6 \%$, las actividades de servicios sociales, comunales y personales con un $6,9 \%$ y la agricultura, ganadería, caza, silvicultura y pesca con un 6,8\%. Otros componentes rezagados son servicios financieros con un $4,5 \%$, y el industrial manufacturero solo con el $2,6 \%$.

La inflación del departamento (departamento, 2013), para el mes de julio 2013, se ubica sobre el promedio nacional con un 1,88 producto en niveles superiores en la categorías de alimentos y vestuario.

Sin analizar el sector petrolero ya que este tendrá un mercado cuya demanda permanente, veremos otros sectores como el industrial el cual esa compuesto principalmente por: molinería y sus productos; frutas, legumbres aceites y grasas.

Industria 2011

Producción industrial

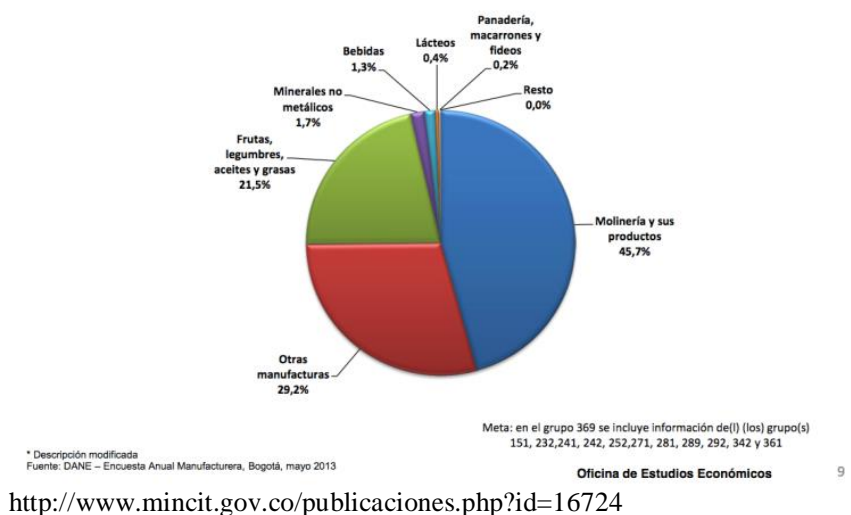

El sector agrícola presenta los siguientes resultados y conformación:

Sector agrícola 2011

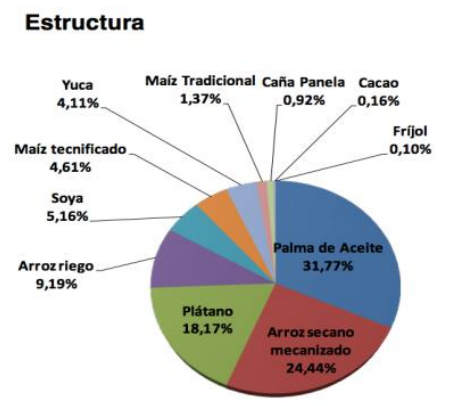

La actividad agrícola del departamento se concentra en 4 productos, en los cuales, ocupa los siguientes lugares en el total nacional:

Arroz secano mecanizado:1er puesto. Palma de aceite: 1er puesto.

Plátano: $4^{\circ}$ puesto.

Arroz riego: $5^{\circ}$ puesto.

En otros productos, se destaca como: - El 1er. productor de soya.

- El $5^{\circ}$. productor de maiz tecnificado.

Ver Anexo A

ra y Desarrollo Rural. Anuario Estadistico 201 .

http://www.mincit.gov.co/publicaciones.php?id=16724

En cuanto a la producción de frutas (departamento, 2013) se concentra en tres productos como son: los cítricos, la patilla, guayaba, piña y maracuyá. La producción pecuaria del departamento lo compone en su orden el ganado bovino, y especie equina.

Desde el punto de vista productivo (departamento, 2013), el departamento presenta oportunidad en la utilización de recursos naturales para la explotación agrícola y pecuaria, de esta manera tomando apartes del análisis del diagnóstico General del Meta, (Llanos, 2005), tenemos que en el departamento sobresale la producción piscícola como aprovechamiento de recursos del territorio. Existe potencial en la producción de frutas, plantas medicinales, productos silvícolas, zoocría y agroforestería. Además de contar con una gran riqueza hídrica, que desde los páramos hasta las sabanas de la Orinoquia. Su posición con acceso terrestre al mercado de Bogotá y la proyección de 
ejes viales y fluviales con Venezuela y el pacífico ofrece un abanico de desarrollo a futuro, no obstante las limitaciones estructurales existentes y la escasa tradición industrial.

Oportunidades para el departamento del Meta. La Alianza del Pacífico, como los demás acuerdos o tratados de comercio, trae al país enormes oportunidades de negocios, ya que se establecen preferencias comerciales de entrada a los mercados, es decir, se tiene mayor libertad de ingreso a nuevos mercados, el reto está en las condiciones internas del país y del departamento para generar un ambiente y condiciones socioeconómicas y jurídicas, para las empresas que deseen incursionar al mercado global. Las oportunidades concretas se orientan al servicio de turismo y productos no tradicionales como frutas, cítricos, cárnicos, y artesanías. Esto teniendo en cuenta que a través de los países miembros se podrá acceder a un mayor número de países a nivel mundial. El Departamento del Meta, debe aprovechar los recursos abundantes que posee para el aprovechamiento de la Alianza.

El informe Monitor, entregado al país este año, establece imperativos estratégicos para Colombia como son:

- Apoyo a la empresa y su proceso de creación

- Desarrollar centro de innovación

- Fortalecer la demanda local y la relación con los oferentes y demandantes

- Educación y capacitación en comercio

- Crear un consejo de competitividad

- Apoyar la creación de una prensa económica

- Apoyo a la formación de recursos humanos especializados

- Y vender la marca Colombia

Igualmente, plantea el informe dado por el Sr. Michael Porter, que los sectores más importantes para Colombia en los que puede desarrollar ventajas competitivas son: Bienes de capital, jugos de frutas, flores, cuero, artes gráficas y los textiles. De lo anterior, el país ha orientado esfuerzos hacia otros aspectos.

El departamento del Meta debe enfocar sus esfuerzo al aprovechamiento de oportunidades como: Las regalías petroleras e invertir en la formación de capitales productivos; La utilización de sus tierras aunque en su mayoría poco fértiles, pero con tratamientos complementarios puede aumentar sus niveles de producción, Aumentar la productividad agropecuaria de las zonas de mayor productividad como es el área del piedemonte y utilizando recursos abundantes. Recuperar la navegabilidad por el rio Meta, para disminuir los costos de transporte, y mejorar la posibilidad de exportar a través de Venezuela. Explotar aún más el servicio de gobierno en línea, ya que es un país con ventaja en este campo

\section{RETOS}

Incremento de las condiciones de infraestructura y logística del departamento, como mejorar las condiciones viales, concretar el proyecto de aeropuerto internacional, y la navegabilidad del rio Meta como ruta de comercio a Venezuela y atlántico. Lo anterior a fin de disminuir las desventajas geográficas.

El problemas social de país ha generado un desplazamiento de población a las urbes, lo que implica que la necesidad de asegurar los derechos de propiedad como factor de confianza y seguridad.

Definir un plan de desarrollo rural articulado con actividades de conservación del medio.

El diseño e implementación de políticas agropecuarias y económicas que permita integrar el territorio departamental físico, económico y legalmente a las nuevas condiciones comerciales del país y del mundo.

\section{DESAFÍOS}

El mayor desafío para el departamento y el país, es alcanzar un mayor nivel de integración económica con el mundo a través de los diferentes tratados comerciales.

Que el crecimiento económico genera un mayor nivel de equidad en la distribución de la riqueza

Finalmente al escenario propicio de competitividad que se espera debe ofrecer el Estado, en lo relacionado a políticas estatales de incentivo $\mathrm{y}$ garantías para el desarrollo de las actividades productivas, las empresas del Meta se enfrentan a retos internos aún más complejos y tienen que ver con la cultura organizacional. Es decir:

1. La mentalidad de muchos gerentes dejar de ver que Bogotá puede ser su destino de ventas, y tomar la decisión de "salir" a buscar nuevos mercados para sus productos o servicios, ya que 
los procesos, requisitos o condiciones son cosas que se deben cumplir.

2. El producir alimentos, podríamos afirmar que tienen mercado en cualquier país, el reto radica en ofrecer productos con valor agregado lo que generará mayores niveles de utilidad. Innovación en el producto.

3. Buscar de manera permanente la innovación en los procesos administrativos, productivos o de ventas a fin de reducir costos, obtener mayores niveles de eficiencia, y establecer una ventaja competitiva en costos.

4. Al establecer un mercado y entrar en él, implica estar buscando un nuevo nicho o tomar decisiones de diversificación de producto.

5. Dar el primer paso, en el proceso de indagar, preguntar y decidir buscar nuevos mercados, ya que en la actualidad existen buenas fuentes de información y capacitación en el proceso de querer exportar.

Actualmente el país posee más desventajas competitivas que van desde lo legal, tributario, hasta la infraestructura, lo que impacta fuertemente en los costos de producción o distribución al mercado objetivo, lo que implica que las empresas metenses, aparte de tratar de superar estas barreras o complicaciones internas, deben afrontar decisiones de tipo administrativo de competencia no sólo como empresas locales o nacionales, sino con empresas internacionales.

Podría afirmar que la falta de externalizar - en la posibilidad de utilizar este término - los mercados de las empresas metenses se ha debido a dos factores específicas, como son: La comodidad de operar en el mercado local y de influencia directa y la abundancia de recursos de la región, basado en la premisa "en la escasez y adversidad, surgen la innovación y superación".

Luego de más de 20 años de darnos la bienvenida al futuro, podemos ver que hemos hecho poco, tanto el gobierno como empresarios especialmente los metenses, para aprovechar las ventajas que nos brinda el tener el mercado mundial al alcance de nuestras manos.

\section{BIBLIOGRAFÍA}

Pacifico, A. (02 de 10 de 2013). http://alianzapacifico.net.

Obtenido de

http://alianzapacifico.net:

http://alianzapacifico.net/wp-

content/uploads/2013/09/Cartilla-Versión-para-

impresión-AP.pdf

El Espectador. (08 de 08 de 1990). Apartes del discurso de posesión del Dr. Cesar Gaviria Trujillo. pág. 13A.

Daniels, J. (2010). Negocios Internacionales. México, México: Prentice Hall.

Lora, E. (24 de 08 de 2013). El reto exportador. Dinero.com.

República, B. d. (07 de 10 de 2013). http://www.banrepcultural.org. Obtenido de Banco de la República: http://www.banrepcultural.org/blaavirtual/ayudadetar eas/economia/econo20.htm

Colombia, E. s. (2013). Banco de la República. Recuperado el 01 de 10 de 2013, de http://www.banrep.gov.co:

http://www.banrep.gov.co/sites/default/files/publicac iones/archivos/eser_51_suroriente_2013.pdf

Duque, G. El eje Urabá-Tribugá: La salida a los océanos en Colombia. Universidad Nacional de Manizales, Manizales, Manizales.

Pacífico, C. A. (05 de 10 de 2013). Alianza Pacifico. Recuperado el 05 de 10 de 2013, de http://alianzapacifico.net: http://alianzapacifico.net departamento, P. e. (2013). http://www.mincit.gov.co. Recuperado el 01 de 10 de 2013, de http://www.mincit.gov.co: http://www.mincit.gov.co/publicaciones.php?id=167 24

Llanos, U. d. (2005). Diagnóstico General del Meta. Diagnóstico, Universidad de los Llanos, Instituto de investigaciones de la Orinoquia - IIOC, Villavicencio.

Competitividad, C. P. (2012). Informe Nacional de Competitividad 2012-2013. Consejo Privado de Competitividad. Bogotá: Zetta Comunicadores. 